\title{
On Diffraction and JIMWLK Evolution
}

\author{
Michael Lublinsky \\ State University of New York - Department of Physics and Astronomy \\ Stony Brook NY 11794-3800, USA
}

\begin{abstract}
A systematic approach towards description of semi-inclusive processes at low $x$ and with multiple rescatterings taken into account is highlighted. We discuss diffractive processes and their evolution with respect to relevant rapidity intervals.
\end{abstract}

This talk is based on Ref. [2].

- We develop a general formalism to address semi-inclusive processes at high energies and including multiple rescatterings. Part of formalism is independent of underlying high energy evolution. However, most of applications considered are within DIS framework and assume BK-JIMWLK evolution.

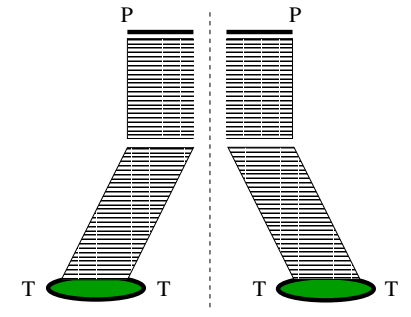

(a)

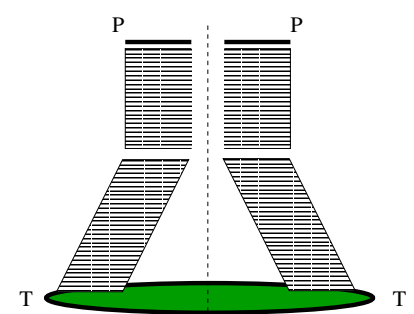

(b)

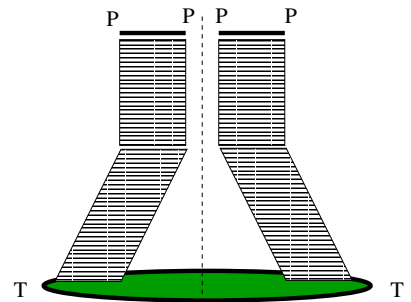

(c)

Figure 1: Diffraction and fan diagrams

- We apply our general formalism to High energy diffractive processes. We attempt to derive results not relying on the dipole (large $N_{c}$ and target factorization) approximation. We reproduce and extend the result of Ref. [3] for the process of projectile diffraction with target scattered elastically. We also obtain results for projectile diffraction with target diffracting in a small rapidity interval and elastic scattering.

- We consider high energy diffraction with multiple gaps. For various diffractive processes we derive evolution equations with respect to total rapidity and gap(s).

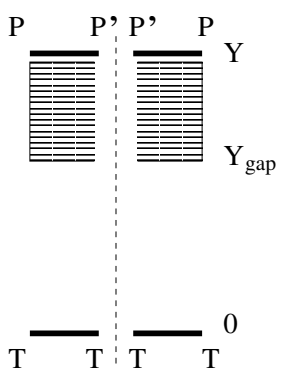

(a)

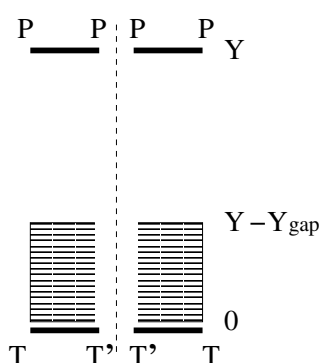

(b)

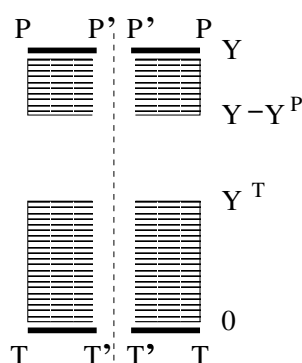

(c)

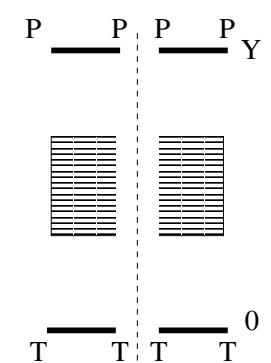

(d)

Figure 2: Various types of diffractive processes 
Let me flash the formalism which is based on the evolution of hadronic wavefunction. Hadron wave function in the gluon Fock space is

$$
|\Psi\rangle=\Psi\left[a_{i}^{\dagger a}(x)\right]|0\rangle \quad|\Psi\rangle=|v\rangle
$$

After rapidity evolution the evolved wave function becomes

$$
\left|\Psi_{i n}\right\rangle=\Omega_{Y}(\rho, a)|v\rangle ; \quad|v\rangle=|v\rangle \otimes\left|0_{a}\right\rangle
$$

Here $\Omega$ is the most general evolution kernel. It is known for arbitrary dense hadron [4]. We will however concentrate on the most simple case of dilute hadron. In this limit, $\Omega$ reduces to the gluon cloud operator

$$
\left.C_{Y} \equiv \Omega_{Y}(\rho \rightarrow 0)=\operatorname{Exp}\left[i \int d^{2} z b_{i}^{a}(z) \int_{\Lambda}^{e^{Y} \Lambda} \frac{d k^{+}}{\pi^{1 / 2}\left|k^{+}\right|^{1 / 2}}\left[a_{i}^{a}\left(k^{+}, z\right)+a_{i}^{\dagger a}\left(k^{+}, z\right)\right)\right]\right] .
$$

with the classical WW field

$$
b_{i}^{a}(z)=\frac{g}{2 \pi} \int d^{2} x \frac{(z-x)_{i}}{(z-x)^{2}} \quad \rho^{a}(x)
$$

The projectile's gluon scattering of a dense target has the eikonal propagator given by the Wilson line

$$
S(x)=\mathcal{P} \exp \left\{i \int d x^{-} T^{a} \mathbf{A}_{t}^{a}\left(x, x^{-}\right)\right\} .
$$

with $A_{t}$ characterizing the target external field. The evolution of the diagonal element of the $S$-matrix operator $\Sigma^{P} \equiv\left\langle\Psi_{\text {out }} \mid \Psi_{\text {in }}\right\rangle$ reads

$$
\partial_{Y} \Sigma^{P}=-H^{J I M W L K} \Sigma^{P} ; \quad \quad H^{J I M W L K}=\int_{z} Q_{i}^{a}(z) Q_{i}^{a}(z)
$$

where we introduced the gluon production (and scattering) amplitude

$$
Q_{i}^{a}(z)=g \int_{x} \frac{(x-z)_{i}}{(x-z)^{2}}\left[J_{L}^{a}(x)-S^{a b}(z) J_{R}^{b}(x)\right]
$$

The generators of the left/right color rotations are Lie derivatives

$$
J_{R}^{a}(x)=-\operatorname{tr}\left\{S(x) T^{a} \frac{\delta}{\delta S^{\dagger}(x)}\right\}, \quad J_{L}^{a}(x)=-\operatorname{tr}\left\{T^{a} S(x) \frac{\delta}{\delta S^{\dagger}(x)}\right\}
$$

This operator is visualized in Fig. 3.

Now we turn to discussion of semi-inclusive reactions. The system emerges from the collision at $t=0$ and keeps evolving to the asymptotic time $t \rightarrow+\infty$, at which point the measurement of an observable $\hat{\mathcal{O}}$ is made

$$
\langle\hat{\mathcal{O}}\rangle=\left\langle v\left|\Omega_{Y}^{\dagger}\left(1-\hat{S}^{\dagger}\right) \Omega_{Y} \hat{\mathcal{O}} \Omega_{Y}^{\dagger}(1-\hat{S}) \Omega_{Y}\right| v\right\rangle
$$

We find it convenient to introduce two targets - one for the amplitude $S$ and another one for its conjugate $\bar{S}$. In the end of our computation we set $S=\bar{S}$.

$$
\mathcal{O}_{Y}[S, \bar{S}]=\left\langle P_{v}\left|\Omega_{Y}^{\dagger}\left(1-\hat{S}^{\dagger}\right) \Omega_{Y} \hat{\mathcal{O}} \Omega_{Y}^{\dagger}(1-\hat{\bar{S}}) \Omega_{Y}\right| P_{v}\right\rangle
$$




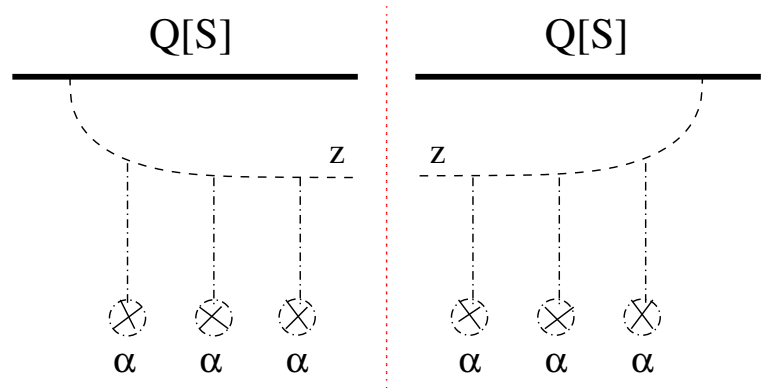

Figure 3: The operator $Q$

High energy evolution of the observable is given by the following equation

$$
\frac{d \mathcal{O}_{Y}[S, \bar{S}]}{d Y}=\lim _{\Delta y \rightarrow 0} \frac{\mathcal{O}_{Y+\Delta y}[S, \bar{S}]-\mathcal{O}_{Y}[S, \bar{S}]}{\Delta y}=-H_{3}[S, \bar{S}] \mathcal{O}_{Y}[S, \bar{S}]
$$

Here the Hamiltonian $\mathrm{H}_{3}$ (first introduced in [5])

$$
\begin{aligned}
& H_{3}[S, \bar{S}] \equiv H_{1}[S]+H_{1}[\bar{S}]+2 \int_{z} Q_{i}^{a}(z,[S]) Q_{i}^{a}(z,[\bar{S}]) \\
& H_{1}[S] \equiv H^{J I M W L K}[S]=\int_{z} Q_{i}^{a}(z,[S]) Q_{i}^{a}(z,[S]), \quad H_{2}[S, \bar{S}] \equiv H_{1}[S]+H_{1}[\bar{S}]
\end{aligned}
$$

is illustrated in Fig. 4. The Hamiltonian $\mathrm{H}_{2}$ appears in diffractive processes and is responsible for evolution through a rapidity gap. The Hamiltonian $H_{3}$ is presumably the answer to properly formulated question of generalization of AGK cutting rules to QCD.

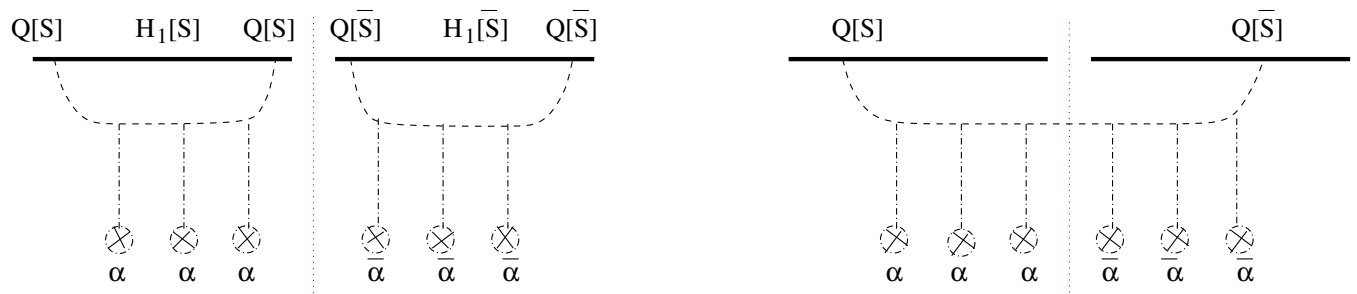

Figure 4: The Hamiltonian $H_{3}$

Having introduced the Hamiltonians we can introduce associated evolution operators:

$$
U_{Y_{1}-Y_{2}}^{3}=\operatorname{Exp}\left[-H_{3}\left(Y_{1}-Y_{2}\right)\right] \quad U_{Y_{1}-Y_{2}}^{2}=\operatorname{Exp}\left[-H_{2}\left(Y_{1}-Y_{2}\right)\right]
$$

Thus a formal solution for inclusive diffraction with multiple gaps and multiple rescatterings (Fig. 5)

$$
\sigma^{d i f f} \sim \int D S D \bar{S} W^{t}[S] \delta(S-\bar{S}) U_{Y_{0}-Y_{n}}^{3} U_{Y_{n}-Y_{n-1}}^{2} \cdots U_{Y_{1}-Y_{2}}^{2} U_{Y-Y_{1}}^{3} \Sigma^{p}[S, \bar{S}]
$$


This expression is quite complex and of little use. Things become less formal and more useful when passing to the dipole degrees of freedom

$$
s_{x, y}=\frac{1}{N} \operatorname{tr}\left[S_{F}(x) S_{F}^{\dagger}(y)\right]
$$

We need to remember, however, that the factorization

$\langle s(x, y) s(u, v)\rangle_{T}=\langle s(x, y)\rangle_{T}\langle s(u, v)\rangle_{T}$

is not always valid. This is very important in order to include target diffractive states.

For processes involving transverse momentum transfer, a quadrupole operator is also in need

$q_{x, y, u, v}=\frac{1}{N} \operatorname{tr}\left[S_{F}(x) S_{F}^{\dagger}(y) S_{F}(u) S_{F}^{\dagger}(v)\right]$

Fortunately, no other higher multiplet operators emerge, if the projectile at rest is made only out of dipoles.

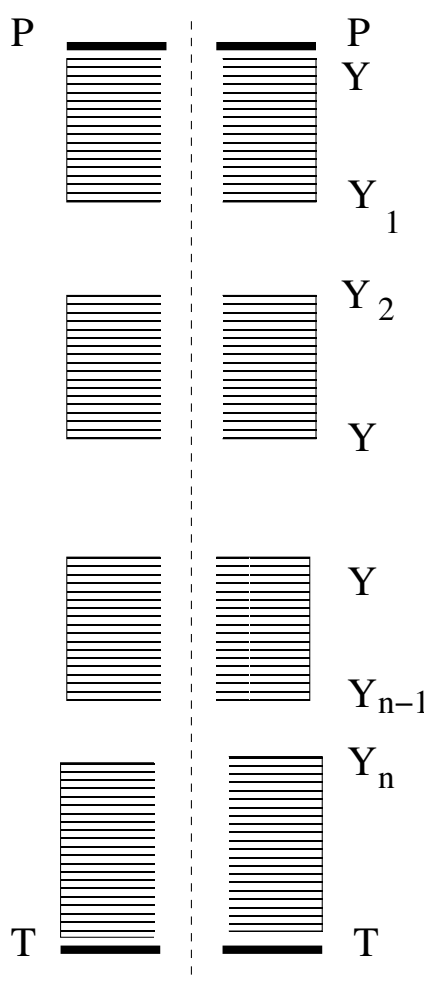

Figure 5: Diffraction with multiple gaps

\section{Acknowledgments}

Everything which is reported above has been done in collaboration with Alex Kovner. Many thanks to Alex for making hard work into a joy.

\section{References}

[1] Slides: http: //indico. cern. ch/contributionDisplay $\cdot$ py? contribId=59\&sessionId=7\&conf Id=9499

[2] A. Kovner and M. Lublinsky, JHEP 0611083 (2006); A. Kovner, M. Lublinsky and H. Weigert, Phys. Rev. D74 114023 (2006).

[3] Y. V. Kovchegov and E. Levin, Nucl. Phys. B 577221 (2000).

[4] A. Kovner, M. Lublinsky and U. Wiedemann, JHEP 0706075 (2007).

[5] M. Hentschinski, H. Weigert and A. Schafer, Phys. Rev. D73 051501 (2006). 